\title{
Negative refraction and imaging beyond the diffraction limit by a two-dimensional left-handed metamaterial
}

\author{
Ekmel Ozbay $^{\mathrm{a}, \mathrm{b}, *}$, Koray Aydin ${ }^{\mathrm{a}}$ \\ ${ }^{a}$ Nanotechnology Research Center-NANOTAM, Department of Physics, Bilkent University, Bilkent, 06800 Ankara, Turkey \\ ${ }^{\mathrm{b}}$ Department of Electrical and Electronics Engineering, Nanotechnology Research Center - NANOTAM, \\ Bilkent University, Bilkent, 06800 Ankara, Turkey
}

Received 15 June 2007; received in revised form 30 August 2007; accepted 9 September 2007

Available online 14 September 2007

\begin{abstract}
We report our experimental results on two-dimensional left-handed metamaterials (LHM) exhibiting negative refraction and subwavelength imaging. Transmission and reflection spectra of LHM are studied and a left-handed transmission band is observed at the frequencies where both dielectric permittivity and magnetic permeability are negative. Impedance matching is verified both with the experiments and simulations. The two-dimensional LHM structure is verified to have a negative refractive index. We employed three different methods to observe negative refraction; refraction through prism-shaped LHM, beam shifting method, and phase shift experiments. We further demonstrated subwavelength imaging and resolution using LHM superlenses. The effect of thickness on the resolving power is investigated experimentally.
\end{abstract}

(C) 2007 Elsevier B.V. All rights reserved.

PACS : 41.20.Jb; 78.20.Ci; 42.70.-a; 81.05.-t

Keywords: Metamaterial; Left-handed material; Negative refraction; Subwavelength imaging; Superlens

\section{Introduction}

In recent years, there has been a burgeoning interest in rapidly growing field of metamaterials due to their unprecedented properties unattainable from ordinary materials. The idea of conceptual materials exhibiting negative values of permittivity $(\varepsilon)$ and permeability $(\mu)$ were first discussed by Veselago in his seminal paper [1]. Inspired by the Pendry's encouraging ideas for obtaining negative permittivity [2] and negative permeability [3] at microwave frequencies, Smith et al. succeeded in designing and demonstrating a

\footnotetext{
* Corresponding author at: Nanotechnology Research CenterNANOTAM, Bilkent University, Bilkent, 06800 Ankara, Turkey. Tel.: +90 312 2901966; fax: +90 3122901015 .

E-mail address: ozbay@bilkent.edu.tr (E. Ozbay).
}

composite metamaterial with properties that are not found in natural materials [4]. Left-handed electromagnetism and negative refraction are achievable with artificially structured metamaterials exhibiting negative values of permittivity and permeability simultaneously at a certain frequency region. Extensive numerical simulations [6-11] and experimental measurements [12-22] are performed to understand the underlying physical mechanism of left-handed metamaterials (LHM). Another exciting physical behaviour of negative-index materials is that they can restore the amplitude of evanescent waves and therefore enable subwavelength focusing [23].

In this study we review our research efforts in the field of metamaterials and report on the transmission, reflection, refraction and imaging properties of twodimensional (2D) left-handed metamaterials composed 
of split-ring resonators and thin wire media. A lefthanded transmission band is observed within frequency region where both $\varepsilon$ and permeability $\mu$ are negative. Refractive index is verified to be negative at these frequencies by using three independent measurement methods that are refraction from prism-shaped LHMs, from slab-shaped LHMs and phase measurements. Consequently, imaging and resolution beyond the diffraction limit is observed for a 2D LHM superlens.

\section{Two-dimensional left-handed metamaterial}

The LHM studied in this work consists of the periodic arrangement of SRR and thin wire arrays. Split-ring resonator and wire patterns are fabricated on the front and back sides of FR4 printed circuit boards, respectively. Fig. 1(a) shows a schematic picture of 2D LHM. Shaded region is the unit cell of the 2D LHM. The parameters of the SRR structure could be found elsewhere [14]. The length and width of the continuous thin wire structures are $l=19 \mathrm{~cm}$, and $w=0.9 \mathrm{~mm}$. The left-handed material is composed of $N_{\mathrm{x}}=40, N_{\mathrm{y}}=20$ layers. Number of layers along the propagation direction $\left(N_{\mathrm{z}}\right)$ is varied for different type of measurements. The directions are provided in Fig. 1(a) and $z$ direction is the propagation direction as

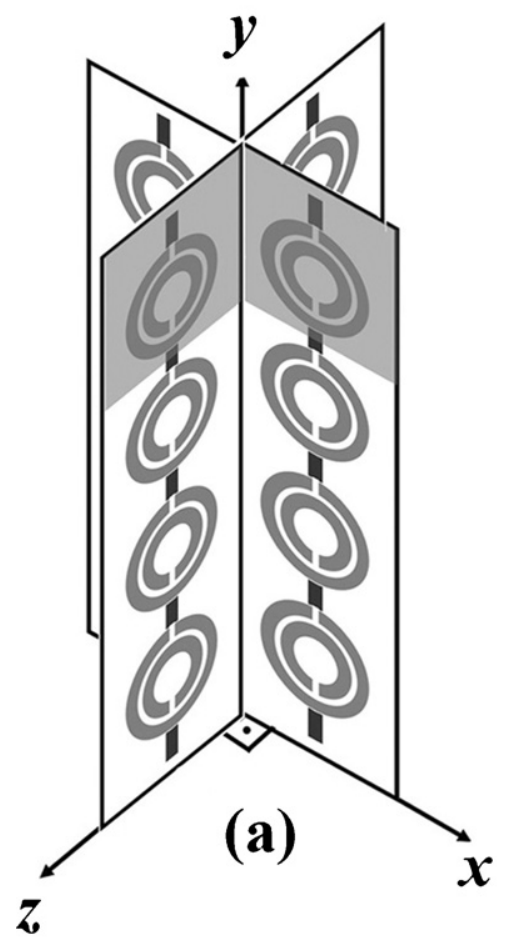

could be seen in Fig. 1(b). The lattice constants are equal on all directions $a_{x}=a_{y}=a_{z}=9.3 \mathrm{~mm}$ [16].

We measured transmission and reflection spectra of 2D LHMs by using HP 8510C network analyzer system that is capable of measuring $S$ parameters, transmission and reflection phase and group delay. In the measurements two horn antennae are connected to the network analyzer, one acts as a transmitter and the other one detects the transmitted or reflected signal. The measurements are performed for LHMs with two different lengths, i.e. number of layers along the propagation direction. Fig. 2(a) shows the measured transmission (black line) and reflection (red dashed line) spectra of LHM with $N_{z}=3$. In a previous work, the negative permeability band gap of SRR structure was observed between 3.60 and $4.05 \mathrm{GHz}$ [16]. On the other hand, the plasma frequency of wire medium was found as $8.0 \mathrm{GHz}$. Therefore, one should observe a transmission band within the frequency region where both permittivity and permeability are negative. It is worthy of note that the effective plasma frequency reduces down to $5.4 \mathrm{GHz}$, when wire arrays are combined with the SRR arrays [14]. As seen in Fig. 2(a), a transmission band is observed between 3.65 and $4.05 \mathrm{GHz}$, where the effective permeability and
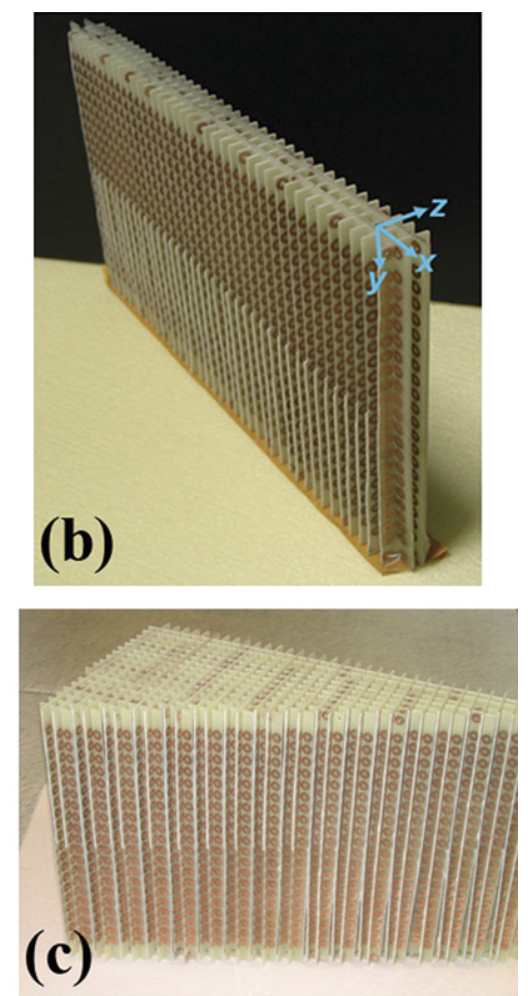

Fig. 1. (a) Schematic drawing of a two-dimensional LHM composed of split-ring resonators on the front and wires at the back of printed circuit board. Photographs of (b) three layer 2D slab-shaped LHM, and (c) prism-shaped LHM. 

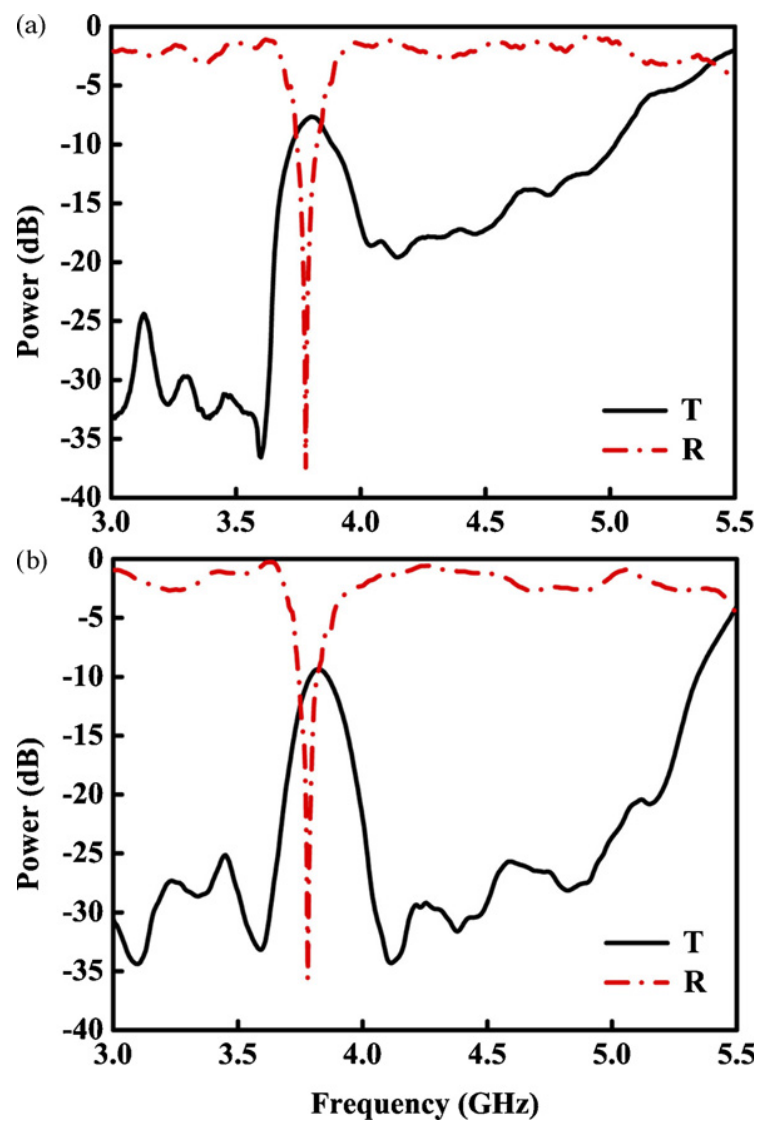

Fig. 2. Measured transmission (black line) and reflection (red dashed line) spectra of (a) three layer, and (b) five layer two-dimensional lefthanded metamaterial.

effective permittivity of LHM are simultaneously negative. The peak value within this transmission band is $-7.6 \mathrm{~dB}$. The peak value reduces to $-9.9 \mathrm{~dB}$ for LHM with $N_{z}=5$ layer as plotted with black line in Fig. 2(b). The reduction in the transmission peak could be attributed to the increased loss that is caused by the increased amount of metal and lossy substrate in the system. The highest transmission for LHM structures are reported by Aydin et al. with a $-1.3 \mathrm{~dB}$ peak value for five layers along propagation direction [14]. The structure was a 1D LHM. The SRR-wire boards are introduced into the system perpendicular to the propagation direction, which reduced the transmission value down to $-9.9 \mathrm{~dB}$. Three-dimensional structures will have even higher losses and lower transmission.

The reflection spectra of three and five layer LHMs are given with dashed red lines in Fig. 2(a) and (b). For both structures, we observed a dip in the reflection spectra at $3.78 \mathrm{GHz}$. The reflection is very low around $-35 \mathrm{~dB}$ meaning that the incident EM waves do not face significant amount of reflection at the LHM surface. The low reflection from the surface can be attributed to either matched impedance at the interface or to the thickness resonance of the slab. Since the frequency for lowest reflection did not change for three and five layered LHMs, such behaviour could not be due to the thickness resonance.

The effective parameters of the LHM structure are retrieved by using the calculated amplitudes and phases of transmission and reflection. Fig. 3(a) shows the real parts of $\mu$ (black solid line), $\varepsilon$ (red dashed line) and $n$ (dashed-dotted line). $\varepsilon^{\prime}$ and $\mu^{\prime}$ are simultaneously negative between 3.63 and $3.93 \mathrm{GHz}$. Refractive index is also negative at these frequencies. The real part of the impedance, $Z^{\prime}$ (red line) and simulated reflection spectrum (black line) are shown in Fig. 3(b). The impedance is defined as $Z=\sqrt{\mu^{\prime} / \varepsilon^{\prime}}$, therefore impedance matching is obtained when $\varepsilon^{\prime}=\mu^{\prime}$. The minimum reflection in the simulations occurs at $3.74 \mathrm{GHz}$, where $\varepsilon^{\prime}=\mu^{\prime}=-1.8$ (dashed lines in Fig. 2). Expectedly, the impedance of LHM is matched to that of free-space at $3.74 \mathrm{GHz}$, where $Z^{\prime}=1$.
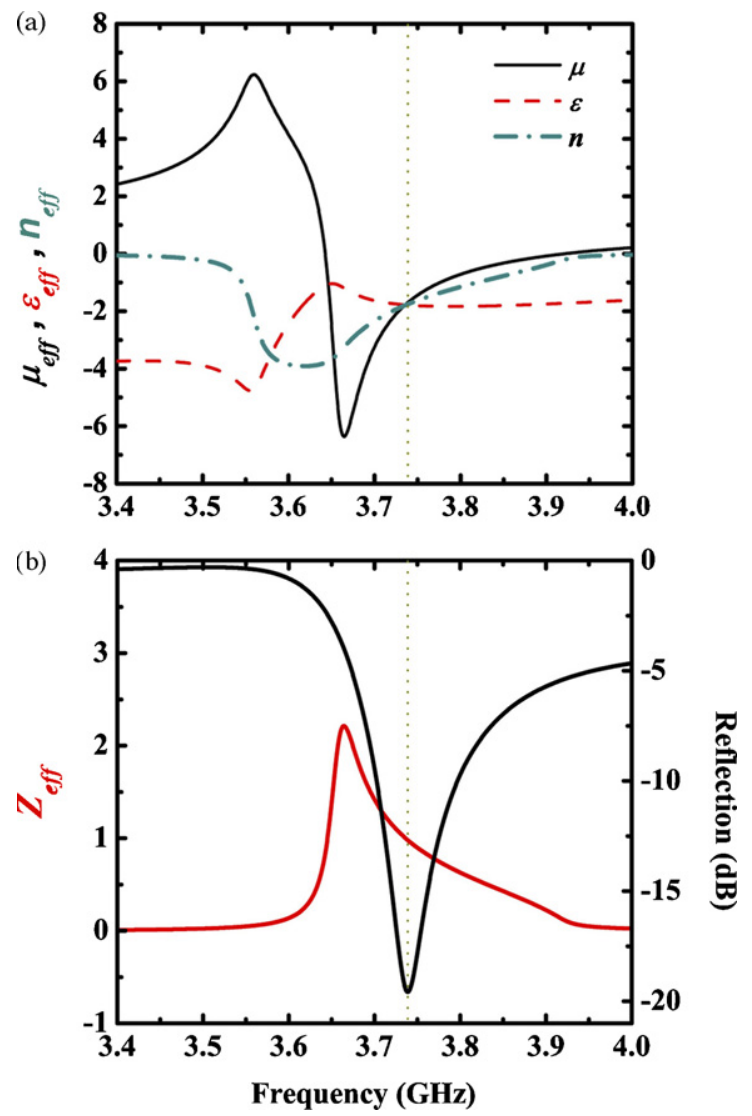

Fig. 3. Retrieved effective parameters of 2D LHM. (a) Real parts of permeability, permittivitiy and refractive index, and (b) reflection spectrum and real part of impedance. 


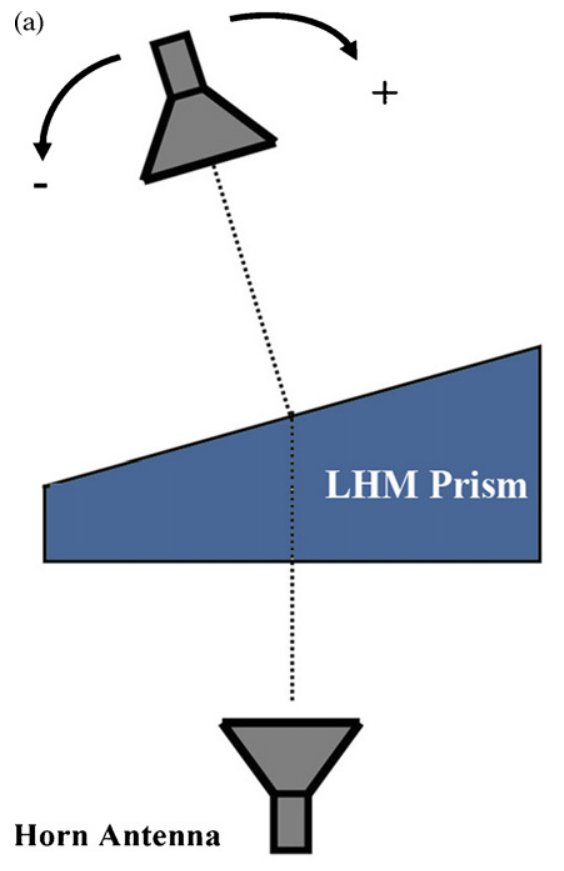

(b)

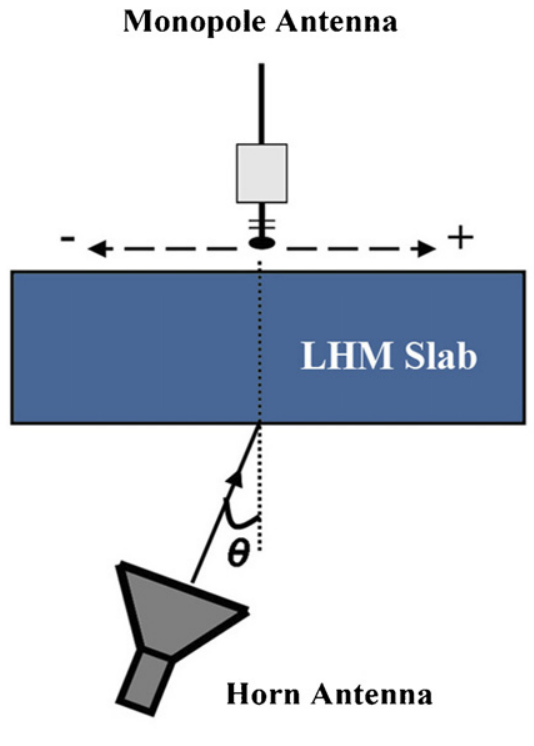

Fig. 4. Schematic drawings of experimental setups for refraction experiments by using (a) beam-shift technique, and (b) prism-shaped LHM.

\section{Experimental observation of negative refraction}

We performed three different and independent experimental measurements to verify that the index of refraction is negative. First we measured refraction through a $2 \mathrm{D}$ prism-shaped left-handed material. We also performed refraction experiments on slab-shaped LHM by using beam-shift method. Finally we performed measurements to observe negative phase shift for LHMs with varying lengths and found that the effective refractive index is negative.

Prism-shaped structures can be used to find the sign and the value of the refractive index $[5,16]$. We constructed a prism-shaped LHM with a prism angle of $\theta=26^{\circ}$ as seen in Fig. 1(c). The schematic drawing of experimental setup can be seen in Fig. 4(a). The first interface of left-handed material is excited with EM waves emanating from the transmitter horn antenna located at a distance of $13 \mathrm{~cm}$ away from the first interface of the prism. The receiver horn antenna is placed $70 \mathrm{~cm}$ away from the second interface of the sample and mounted on a rotating arm to scan the angular distribution of the refracted signal. The angular refraction spectrum is scanned by $\Delta \theta=2.5^{\circ}$ step size.

The measured field intensity at $70 \mathrm{~cm}$ away from the prism is plotted as a function of frequency and refraction angle in Fig. 5. From Snell's law which is given by $n_{\text {eff }} \sin \theta_{\mathrm{i}}=n_{\text {air }} \sin \theta_{\mathrm{r}}$, negative refraction angle could only be possible if the refractive index of the measured sample is negative. This measurement verifies that the refractive index values of the $2 \mathrm{D}$ LHM is negative between 3.73 and $4.05 \mathrm{GHz}$. The angle of the refraction at $3.78 \mathrm{GHz}$ is measured to be $\theta_{\mathrm{r}}=-65^{\circ}$. By using Snell's law, the refractive index is calculated as $n_{\text {eff }}=-2.07 \pm 0.05$ at $3.78 \mathrm{GHz}$. The errors for the

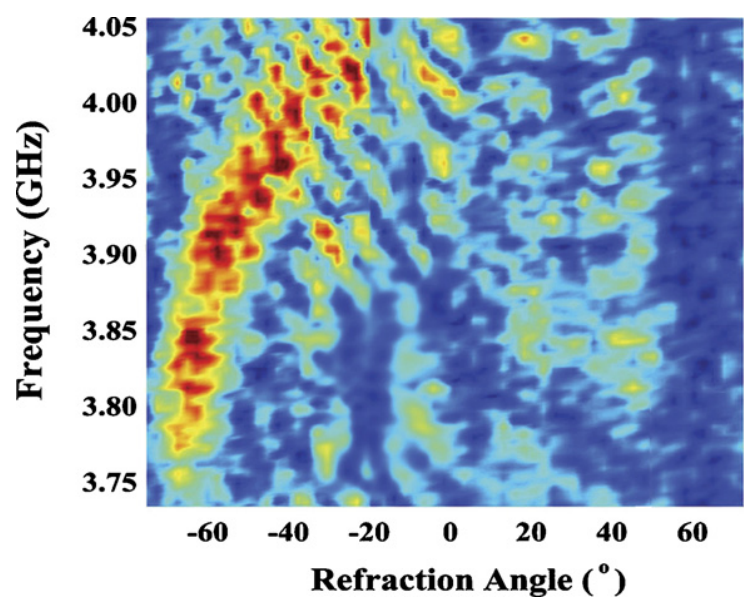

Fig. 5. Measured beam profiles of the EM waves refracted from a 2D prism-shaped LHM as a function of frequency and angle of refraction between 3.73 and $4.05 \mathrm{GHz}$. The beam clearly refracted on the negative side of the normal, indicating a negative refraction behaviour. 


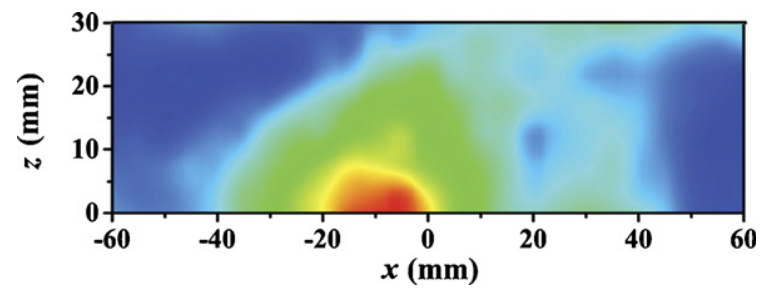

Fig. 6. Measured beam profiles of EM waves refracted from a slabshaped LHM at $3.86 \mathrm{GHz}$.

measured refractive index is due to the finite angular step size of $\Delta \theta=2.5^{\circ}$.

Beam shifting method can also be used to calculate an effective refractive index for a medium $[18,19]$. A slab-shaped LHM sample is excited with a plane wave and the refracted beam profile is determined by scanning the field intensity on the second LHM-air interface. A schematic drawing for the experimental setup is drawn in Fig. 4(b). The transmitter is a horn antenna and placed on the negative side $(-x)$ of the LHM structure with respect to its central axis. The horn antenna is $125 \mathrm{~mm}$ away from the first interface of the LHM slab. The plane wave is sent through the LHM slab with an incident angle of $\theta_{\mathrm{i}}=15^{\circ}$. The intensity distribution of the EM wave is scanned by a monopole antenna mounted to a $2 \mathrm{D}$ scanning table with $\Delta x=\Delta z=2.5 \mathrm{~mm}$ steps. We used a 10 layer LHM in order to observe the shift clearly. The measured refraction spectrum at $3.86 \mathrm{GHz}$ is given in Fig. 6. The incident EM wave has a Gaussian beam profile centered at $x=0$. Therefore, by measuring the shift of the outgoing beam, one can easily deduce whether the structure has a positive or negative index of refraction. As clearly seen in Fig. 6, the center of the outgoing Gaussian beam is shifted to the left side of the center of the incident Gaussian beam, which due to Snell's law, corresponds to negative refraction. The refractive index of LHM calculated from Snell's law $\left(n_{\text {air }} \sin \theta_{\mathrm{i}}=n_{\text {eff }} \sin \theta_{\mathrm{r}}\right)$ as $n_{\text {eff }}=-1.91$.

\section{Verification of negative-index by phase shift experiments}

For materials with a negative refractive index, the phase velocity and energy flow are anti-parallel inside a LHM. Therefore the phase velocity points toward the source. We developed a method to observe the phase shift by measuring the transmission phases for LHMs with varying thicknesses and verified that the phase velocity is negative [16]. We have constructed four different 2D LHM slabs with number of layers $N_{z}=5,6$, 7 and 8. The transmitted phases are plotted in the
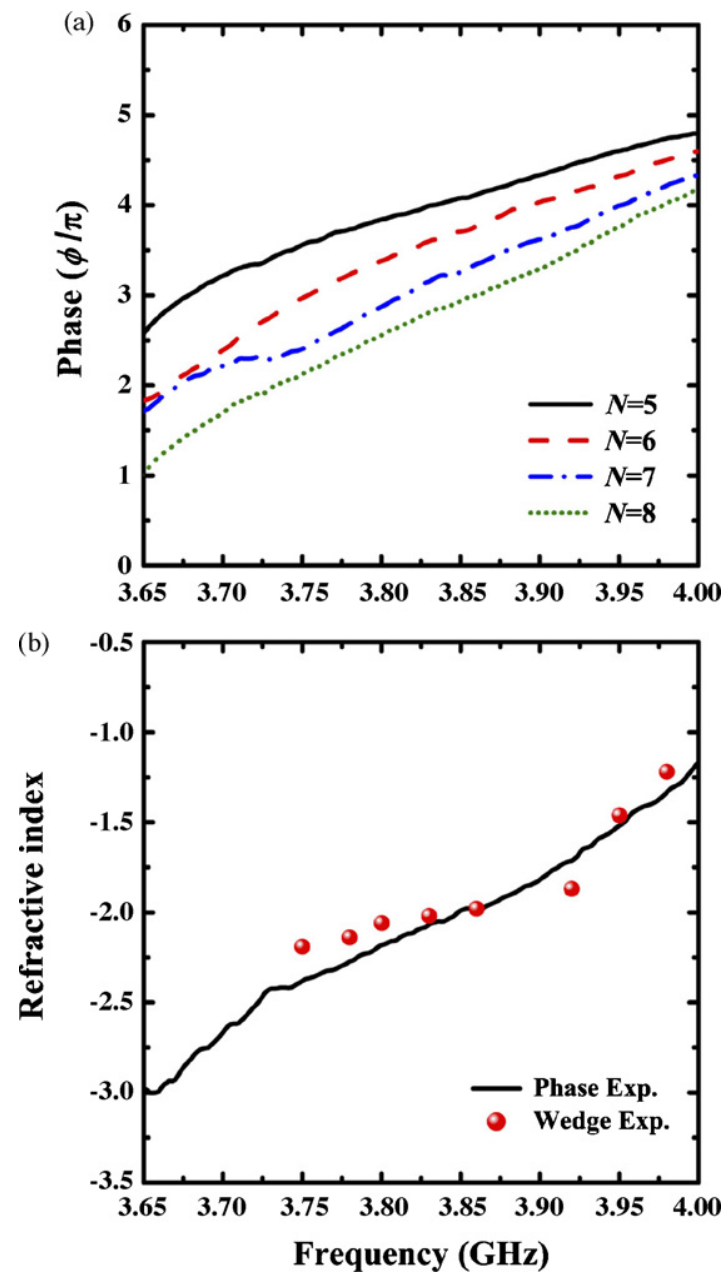

Fig. 7. (a) The measured transmission phase spectra of LHMs with five, six, seven and eight number of layers along the propagation direction. (b) Measured effective refractive indices as a function of frequency that are obtained from the phase shift experiments (black line) and prism experiments (

frequency range $3.65-4.00 \mathrm{GHz}$, which is within the left-handed transmission region.

Fig. 7(a) shows the transmitted phase for different number of layers of LHM slabs. It is evident from the figure that, increasing the number of layers of LHM results in a decrease at the phase of the transmitted EM wave. However, if the material possesses a positive refractive index, one would observe an increase in the transmitted phase with the increasing number of unit cells [16].

The index of refraction in terms of wavelength, phase shift, and change in the length of left-handed material is given by [16]:

$n=\frac{\Delta \Phi}{\Delta L} \frac{\lambda}{2 \pi}$ 
At $f=3.86 \mathrm{GHz}$, the wavelength of the EM wave is $\lambda=7.77 \mathrm{~cm}$. The average phase shift per unit cell $(\Delta L=8.8 \mathrm{~mm}$ ) obtained from the experimental results is $\Delta \Phi=-(0.45 \pm 0.04) \pi$. Inserting these values in (1), index of refraction at $3.86 \mathrm{GHz}$ is found to be $n_{\text {eff }}=-1.98 \pm 0.18$. The error bar is a result of the measured phase difference $( \pm 0.05 \pi)$ between consecutive numbers of LHM layers. The average phase shift and calculated refractive index for the numerical simulations at $3.77 \mathrm{GHz}$ are $\Delta \Phi=-(0.51 \pm 0.04) \pi$, and $n_{\text {eff }}=-2.31 \pm 0.18$.

In Fig. 7(b), we plotted the refractive index values calculated by using the phase shift between the consecutive numbers of LHM layers. The symbol (O) shows the values of measured refractive index obtained from prism experiments at some other frequencies. There is a good agreement between the results obtained from two different methods. We also found index of refraction at $3.86 \mathrm{GHz}$ by using beamshift method as -1.91 . The index of refraction values obtained from the prism and the phase shift experiment are both -1.98 at this frequency. Therefore we have been able to show that the results obtained from three different experiments agree extremely well.

\section{Subwavelength imaging and resolution}

A perfect lens is one of the most important applications of materials with a negative refractive index. The term, perfect lens, was coined by Pendry owing to the ability of such lenses to reconstruct a perfect image by recovering the evanescent components of EM waves [23]. In conventional optics, the lenses are constructed from positive-index materials and require curved surfaces to bring EM waves into focus. Positiveindex lenses suffer from the diffraction limit and can only focus objects with sizes on the order of or greater than a half-wavelength.

The imaging measurements are performed at $3.78 \mathrm{GHz}$, where the reflection is considerably low and the losses due to reflection are negligible. The impedance is matched at $3.78 \mathrm{GHz}$ for perpendicular normal incidence; however the reflection will still affect the performance of LHM superlens for oblique incidence. In the imaging experiments, we employed monopole antennae to imitate the point source. The exposed center conductor acts as the transmitter and receiver and has a length of $4 \mathrm{~cm}(\sim \lambda / 2)$. Firstly, we measured the beam profile in free-space that is plotted in Fig. 8 with a blue dashed line. The full width at half

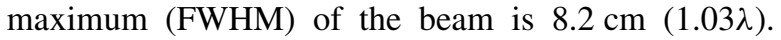
Then, we inserted LHM superlens, and measured the

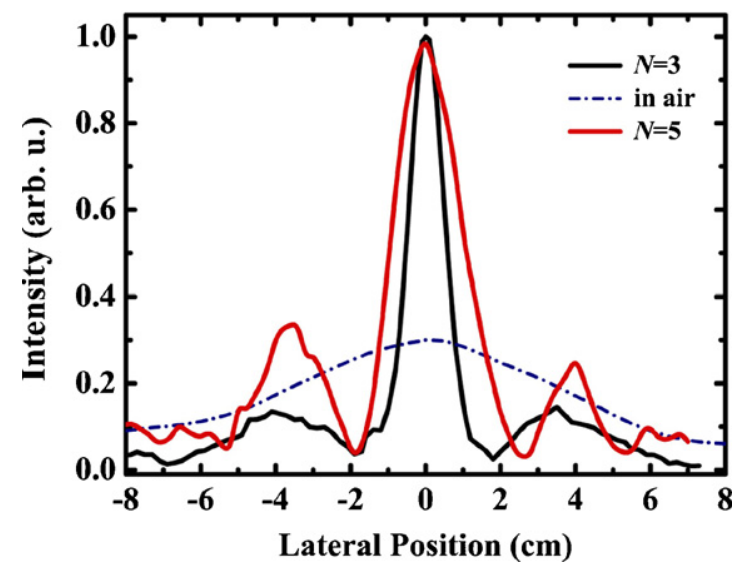

Fig. 8. The measured power distributions at the image plane with three layer (black line) and five layer (red line) LHM superlenses. Blue dashed line corresponds to the power distribution in free-space. Normalized intensity in free-space is multiplied with 0.3 .

spot size of the beam as $0.13 \lambda$, which is well below the diffraction limit (black line in Fig. 8) [24]. The LHM superlens has $40 \times 20 \times 3$ layers along the $x, y$, and $z$ directions. The source is located $d_{\mathrm{s}}=1.2 \mathrm{~cm}$ away from first boundary and the image forms $d_{\mathrm{i}}=0.8 \mathrm{~cm}$ away from second boundary of the superlens. The intensity of the electric field at the image plane is scanned by the receiver monopole antenna with $\Delta x=1 \mathrm{~mm}$ steps. We also performed additional measurements in order to check the influence of the lens thickness on the resolution of the superlens. The thicker LHM superlens has higher losses due to the increased amount of lossy substrate and the metal. Thus, one would expect the resolution to decrease for a thicker superlens. We performed imaging measurements for $N_{z}=5$ layer superlens and the beam profile at the image plane is plotted with a red line in Fig. 8. As seen in the figure, the resolution is decreased and the FWHM of the beam is measured as $0.25 \lambda$.

Since we were able to image a single point source with a subwavelength spot size, we used two point sources separated by distances smaller than a wavelength to obtain subwavelength resolution. The sources are driven by two independent signal generators and the power distribution is detected by using a microwave spectrum analyzer. The frequencies of the sources differ by $1 \mathrm{MHz}$ to ensure that the sources are entirely incoherent. The imaging experiments are performed for two different separation distances between the sources. The measured power distribution of sources, separated by $\lambda / 8$, is plotted by the black line in Fig. 9. As seen in the figure, the peaks of two sources are resolved. The distance between the two peaks is measured as $1.1 \mathrm{~mm}$, whereas the sources are separated by $1.0 \mathrm{~mm}(\lambda / 8)$. The 

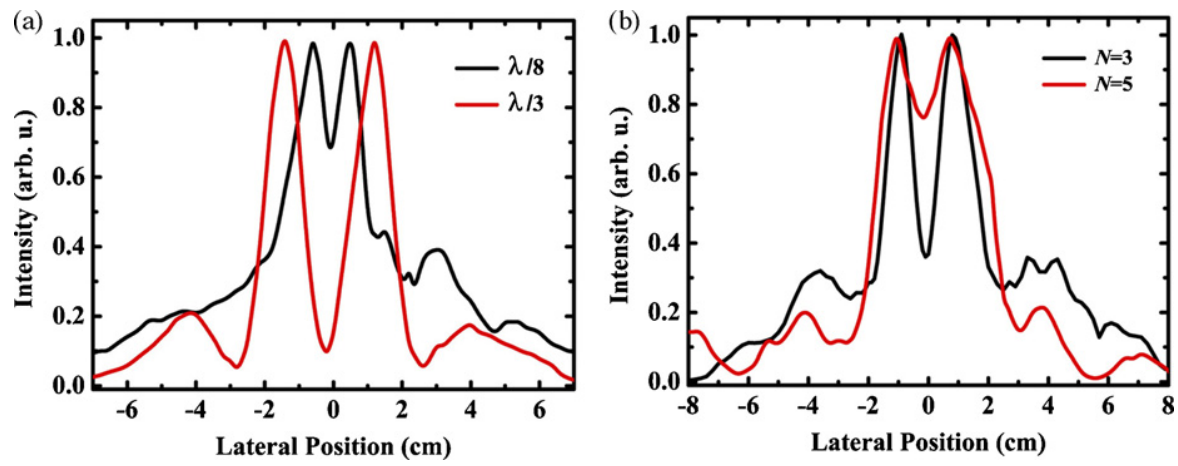

Fig. 9. (a) The measured power distributions for two point sources separated with distances of $\lambda / 8$ (black line), and $\lambda / 3$ (red line). (b) The measured beam profiles for two point sources separated by $\lambda / 5$ from the superlenses with number of unit cells along the propagation direction are $N=3$ (black line) and $N=5$ (red line).

difference could be attributed to the alignment of the LHM superlens with respect to the source and receiver antennas. When the sources are $\lambda / 3$ apart (red line), two peaks are entirely resolved. We also performed experiments by assigning the distances between the sources as $\lambda / 4$ (data not shown) and $\lambda / 5$. The beam profile from the three layer LHM superlens is shown by black line in Fig. 9(b) when the sources are separated by a distance of $\lambda / 5$. We then inserted a thicker LHM superlens with $N=5$ unit cells along the propagation direction. The resulting beam profile is plotted with red line in Fig. 9(b). As clearly observed from Fig. 9(b), the thin superlens has a better resolution compared to the thicker superlens. The presence of losses due to absorption hinders the evanescent wave amplification when thicker lenses are used.

In order to avoid any possible channeling effects, the sources are intentionally not placed at the line of SRRwire boards. Besides, the distances between the sources in the experiments are carefully chosen such that they are not multiples of the lattice constant. However, there is no restriction on the position and the distance between the signals and subwavelength resolution is also achieved when the sources are located in the SRRwire board axes. The lattice constant is on the order of $\lambda /$ 8.5 , which is well below the wavelength and indeed the LHM structure behaves as an effective medium. The periodicity has a significant effect on the resolution of the superlens by limiting the recovery of evanescent components [25].

In the near-field regime, the electrostatic and magnetostatic limits apply, and therefore, the electric and magnetic responses of materials can be treated as decoupled [23]. This in turn brings the possibility of constructing superlenses from materials with negative permittivity or negative permeability. Single-negative lenses can only focus EM waves with one particular polarization. The advantage of using negative-index lenses over negative-permittivity or negative-permeability lenses is that the subwavelength resolution can be obtained for both transverse-electric (TE) and transverse-magnetic (TM) polarization of EM waves. However, our negative-index metamaterial does exhibit negative refraction for single polarization and thus could resolve subwavelength features for that particular polarization. In the near future, we believe that metamaterials exhibiting negative refractive index with circularly polarized incident EM waves would be designed to achieve subwavelength resolution for TE and TM polarizations.

\section{Conclusion}

In conclusion, we have successfully demonstrated a left-handed transmission band for 2D left-handed metamaterial in free-space. Impedance matching condition is satisfied at a certain frequency regime where a sharp dip in the reflection spectra of LHMs is observed. We proved the left-handed behaviour by the numerical simulations and retrieval procedure. Refractive index is calculated to be negative at the frequency region where both permittivity and permeability are negative. 2D negative-index metamaterial has a negative refractive index at frequencies where dielectric permittivity and magnetic permeability are simultaneously negative. We have been able to observe negative refractive index for 2D LHMs by using three different, independent methods. The results obtained from these experiments are in good agreement. Phase shift and therefore phase velocity are shown to be negative. Finally, an impedance-matched, low loss negative-index metamaterial superlens is demonstrated which is capable of resolving subwavelength features with a record-level $0.13 \lambda$ resolution. This is the highest resolution achieved 
from a negative-index metamaterial superlens. Moreover, two sources separated with a distance of $\lambda / 8$ are clearly resolved. The effect of thickness on the subwavelength resolution is also verified, where a thicker superlens substantially reduced the resolution down to $0.25 \lambda$.

\section{Acknowledgments}

We thank I. Bulu and K. Guven for the helpful discussions. This work is supported by the European Union under the projects EU-NoE-METAMORPHOSE, EU-NoE-PHOREMOST, and TUBITAK under Projects Nos. 105E066, 105A005, 106E198 and 106A017. One of the authors (EO) also acknowledges partial support from the Turkish Academy of Sciences.

\section{References}

[1] V.G. Veselago, Engl. Trans. Sov. Phys. Ups. 10 (1968) 509.

[2] J.B. Pendry, A.J. Holden, W.J. Stewart, I. Youngs, Phys. Rev. Lett. 76 (1996) 4773;

J.B. Pendry, A.J. Holden, W.J. Stewart, I. Youngs, J. Phys. Condens. Matter. 10 (1998) 4785.

[3] J.B. Pendry, A.J. Holden, D.J. Robbins, W.J. Stewart, IEEE Trans. Microwave Theory Tech. 47 (1999) 2057.

[4] D.R. Smith, W.J. Padilla, D.C. Vier, S.C. Nemat-Nasser, S. Schultz, Phys. Rev. Lett. 84 (2000) 4184.

[5] R.A. Shelby, D.R. Smith, S. Schultz, Science 292 (2001) 77.
[6] P. Markos, C.M. Soukoulis, Phys. Rev. E 65 (2002) 036622.

[7] N. Katsarakis, Th. Koschny, M. Kafesaki, E.N. Economou, E. Ozbay, C.M. Soukoulis, Phys. Rev. B 70 (2004) 201101.

[8] N. Katsarakis, Th. Koschny, M. Kafesaki, E.N. Economou, C.M. Soukoulis, Appl. Phys. Lett. 84 (2004) 2943.

[9] M. Kafesaki, Th. Koschny, R.S. Penciu, T.F. Gundogdu, E.N. Economou, C.M. Soukoulis, J. Opt. A: Pure Appl. Opt. 7 (2005) S12.

[10] Th. Koschny, M. Kafesaki, E.N. Economou, C.M. Soukoulis, Phys. Rev. Lett. 93 (2004) 107402.

[11] R.S. Penciu, M. Kafesaki, T.F. Gundogdu, E.N. Economou, C.M. Soukoulis, Photon. Nanostruct.: Fundam. Appl. 4 (2006) 12.

[12] H. Daninthe, S. Fotenipolou, C.M. Soukoulis, Photon. Nanostruct.: Fundam. Appl. 4 (2006) 123.

[13] C.G. Parazzoli, R.B. Greegor, K. Li, B.E. Koltenbah, M. Tanielian, Phys. Rev. Lett. 90 (2003) 107401.

[14] K. Aydin, K. Guven, M. Kafesaki, L. Zhang, C.M. Soukoulis, E. Ozbay, Opt. Lett. 29 (2004) 2623.

[15] A. Grbic, G.V. Eleftheriades, Phys. Rev. Lett. 92 (2004) 117403.

[16] K. Aydin, K. Guven, C.M. Soukoulis, E. Ozbay, Appl. Phys. Lett. 86 (2005) 124102.

[17] K. Aydin, K. Guven, N. Katsarakis, C.M. Soukoulis, E. Ozbay, Opt. Express 12 (2004) 5896.

[18] K. Aydin, I. Bulu, E. Ozbay, Opt. Express 13 (2005) 8753.

[19] K. Aydin, E. Ozbay, J. Opt. Soc. Am. B 23 (2006) 415.

[20] K. Guven, K. Aydin, E. Ozbay, Photon. Nanostruct.: Fundam. Appl. 3 (2005) 75.

[21] A. Djermoun, A. De Lustrac, J.-M. Lourtioz, Photon. Nanostruct.: Fundam. Appl. 5 (2007) 21.

[22] K. Aydin, E. Ozbay, Appl. Phys. A 87 (2007) 137.

[23] J.B. Pendry, Phys. Rev. Lett. 85 (2000) 3966.

[24] K. Aydin, I. Bulu, E. Ozbay, Appl. Phys. Lett. 90 (2007) 254102.

[25] D.R. Smith, D. Schurig, M. Rosenbluth, S. Schultz, S.A. Ramakrishna, J.B. Pendry, Appl. Phys. Lett. 82 (2003) 1506. 